$\begin{array}{llllllllllllllllllll}\text { A C T A } & \text { C H E M I C A } & \text { S C A N D I N A V I C A } & 6 & (1952) & 4 & 75 & -493\end{array}$

\title{
Die Dissoziationskonstanten von Hämoglobin und Hämatin und die Pufferkapazität des Blutes
}

\author{
S. K I L P I
}

Chemisches Institut der Universität Helsinki, Finland

\begin{abstract}
Die Säure-Basen-Titrationskurve von Hämoglobin hat zwei deutliche Inflexionspunkte mit Pufferkapazitätsminima ${ }^{1}$, nämlich bei $\mathrm{p}(a \mathrm{H})=5,8$ und bei $\mathrm{p}(a \mathrm{H})=8,7$. Die zwischen diesen Inflexionspunkten wirkenden Dissoziationskonstanten sind leichter bestimmbar als die übrigen, weil die betreffenden Äquivalentpunkte durch die Inflexionspunkte gegeben sind. Diese Konstanten sind auch die in physiologischer Hinsicht wichtigsten, weil beim $p(a \mathrm{H})$ des Blutes $(7,3)$ die Pufferwirkung hauptsächlich von ihnen abhängt. Die Bestimmung der anderen Dissoziationskonstanten ist unsicherer, da nicht nur die betreffenden Inflexionspunkte fehlen, sondern auch in sauren bzw. alkalischen Lösungen Hydrolyse stattfindet, und weil sich in den ersteren bisweilen die Messungen erschwerende Fällungen bilden. Dies war der Fall insbesondere beim Untersuchen von gefälltem, fabriksmässig hergestelltem Hämoglobin ${ }^{2}$. Die nachstehend mitgeteilten Messungsergebnisse sind mit nativem Hämoglobin erhalten worden. Ausserdem wurden die Säure-Basen-Dissoziationskonstanten von Hämin gemessen, weil es von Interesse war, diese Konstanten mit denen des Hämoglobins zu vergleichen.
\end{abstract}

\section{DIE BEREITUNG DER HÄMOGLOBINLÖSUNG}

Die Hämoglobinlösungen wurden aus Menschen-, Pferde- und Rinderblut bereitet. Das frische Blut wurde unmittelbar durch kräftiges Schütteln defibriniert. Darauf wurde das Hämoglobin durch Einleiten von CO in das eisgekühlte Blut in CO-Hämoglobin übergeführt, die Serumlösung nach Zentrifugieren von den roten Blutkörperchen abgesaugt und das Sediment mit $0,1 N$ Natriumchloridlösung dreimal durch Zentrifugieren gewaschen. Das Sediment wurde dann in destilliertes Wasser gebracht, um die Zellwände 
zu zerstören. Nach Zentrifugieren erhielt man so eine weinrote, klare Hämoglobinlösung. Die in bezug auf $\mathrm{NaCl}$ etwa 0,1 normal gemachten Hämoglobinlösungen wurden bei $0-5^{\circ} \mathrm{C}$ aufbewahrt.

Zur Bestimmung der NaCl-Konzentration wurde aus $50 \mathrm{ml}$ Lösung das Hämoglobin durch Koagulieren ausgefällt. Nach Verdünnen des Filtrats auf $200 \mathrm{~cm}^{3}$ wurde in $50 \mathrm{ml}$ das Chlor gravimetrisch bestimmt.

Zur Bestimmung der Konzentration des Hämoglobins in den in obiger Weise bereiteten Hämoglobinlösungen wurden $50 \mathrm{ml}$ der Lösung auf dem Wasserbad eingedampft, bis das Hämoglobin vollständig koaguliert war. Die Lösung wurde von dem ausgefällten Hämoglobin durch einen Filtertiegel abfiltiert und die Fällung mit warmem Wasser gewaschen. Sie wurde bei $100-102^{\circ} \mathrm{C} \mathrm{bzw}$. im Vakuumexsikkator getrocknet und gewogen. Vergleichsweise wurden $50 \mathrm{ml}$ der Hämoglobinlösung auf dem Wasserbad bis zur Trockne eingedampft und bei $100-102^{\circ} \mathrm{C}$ getrocknet. Von der gewogenen Menge wurde dabei die bekannte NaCl-Menge abgezogen. Die verschiedenen Bestimmungen gaben miteinander übereinstimmende Werte für den Hämoglobingehalt.

Der Stickstoffgehalt des getrockneten Hämoglobins wurde durch Verbrennungsanalyse ermittelt.

Die Analysenresultate sind in Tabelle 1 wiedergegeben.

Tabelle 1.

\begin{tabular}{|c|c|c|c|}
\hline $\begin{array}{l}\text { Ursprung des } \\
\text { Hämoglobins }\end{array}$ & $\begin{array}{l}\text { Hämogl.- \% } \\
\text { der Lösung }\end{array}$ & $\begin{array}{c}\% \mathrm{~N} \text { im } \\
\text { Hämoglobin }\end{array}$ & $\begin{array}{c}\text { NaCl-Konz. } \\
\text { Mol/l. }\end{array}$ \\
\hline Menschenblut & 1,176 & 16,20 & 0,1016 \\
\hline Pferdeblut & 0,828 & 16,28 & 0,1019 \\
\hline Rinderblut & 0,520 & 16,16 & 0,1006 \\
\hline " & 0,861 & 15,70 & 0,1011 \\
\hline
\end{tabular}

\section{DIE BERECHNUNG DER DISSOZIATIONSKONSTANTEN}

Die Dissoziationskonstanten wurden aus den potentiometrisch gemessenen $\mathrm{p}(a \mathrm{H})$-Werten der Säure-Basen-Titration berechnet. In den folgenden Gleichungen, die für Konzentrationswerte abgeleitet worden sind, wird statt $\left[\mathrm{aH}^{+}\right.$] die $\mathrm{H}^{+}$-Ionenkonzentration verwendet werden. Der zu deren Berechnung erforderliche Aktivitätskoeffizient wurde nach der Debye-Hückelschen Theorie berechnet (S. 381). 
Der Ubersichtlichkeit halber wurde bei den Berechnungen als Anfangspunkt der Titration der kleinste $\mathrm{p}(a \mathrm{H})$-Wert der Titrationskurve verwendet, der zugleich der Endpunkt beim Titrieren von Hämoglobin mit einer starken Säure ist. [B] bezeichnet im folgenden die Konzentration der zuzufügenden Menge einer starken Base, berechnet von diesem Punkt aus. Dieser Anfangspunkt wurde so gewählt, dass der gemessene $\mathrm{p}(a \mathrm{H})$-Wert an demselben mit dem mit Hilfe der erhaltenen Konstanten für diesen Punkt berechneten $\mathrm{p}(a \mathrm{H})$ Wert übereinstimmt (S. 380).

Die Säure-Basen-Dissoziationskonstanten bzw. die Hydrolysekonstanten der Basen-Kationen ${ }^{3}$ sowie deren Konzentrationen seien bezeichnet:

$$
\begin{aligned}
& K_{1}>K_{2}>K_{3} \ldots \ldots \ldots \\
& C_{1}=\left[\mathrm{HA}_{1}\right]+\left[\mathrm{A}_{1}^{-}\right] \\
& C_{2}=\left[\mathrm{HA}_{2}\right]+\left[\mathrm{A}_{2}^{-}\right] \\
& C_{3}=\left[\mathrm{HA}_{3}\right]+\left[\mathrm{A}_{3}^{-}\right] \\
& ------- \\
& -----
\end{aligned}
$$

Aus den Gleichgewichtsgleichungen:

$$
\begin{aligned}
& \frac{\left[\mathrm{H}^{+}\right]\left[\mathrm{A}_{1}^{-}\right]}{\left[\mathrm{HA}_{1}\right]}=K_{1} \\
& \frac{\left[\mathrm{H}^{+}\right]\left[\mathrm{A}_{2}^{-}\right]}{\left[\mathrm{HA}_{2}\right]}=K_{2} \\
& ------
\end{aligned}
$$

und aus der nach dem Neutralisationsprinzip sich ergebenden Gleichung:

$$
[\mathrm{B}]+\left[\mathrm{H}^{+}\right]=\left[\mathrm{A}_{1}^{-}\right]+\left[\mathrm{A}_{2}^{-}\right]+\ldots .+\left[\mathrm{OH}^{-}\right]
$$

erhält man für die Berechnung von $K$ die Gleichungen:

$$
\left.\begin{array}{c}
K_{v}=\left[\mathrm{H}^{+}\right] \frac{[B]-\sum_{i=1}^{\nu-1}\left[\mathrm{~A}_{i}^{-}\right]-\sum_{i=v+1}^{\mathrm{n}}\left[\mathrm{A}_{i}^{-}\right]+\left[\mathrm{H}^{+}\right]-\left[\mathrm{OH}^{-}\right]}{C-\left\{[\mathrm{B}]-\sum_{i=1}^{\nu-1}\left[\mathrm{~A}_{1}^{-}\right]-\sum_{i=\nu+1}^{\mathrm{n}}\left[\mathrm{A}_{i}^{-}\right]+\left[\mathrm{H}^{+}\right]-\left[\mathrm{OH}^{-}\right]\right\}} \\
{\left[\mathrm{A}_{i}^{-}\right]=\frac{K_{i} C_{i}}{K_{i}+\left[\mathrm{H}^{+}\right]}}
\end{array}\right\}
$$


die sich zur Berechnung der Dissoziationskonstanten durch Annäherungsrechnung eignen. Bei der Berechnung von $K \nu$ mit Hilfe der $\mathrm{p}(a \mathrm{H})$-Werte zwischen den Äquivalentpunkten $v-1$ und $v$ ist:

$$
\begin{aligned}
& {\left[\mathrm{H}^{+}\right]\left\langle\left\langle K_{v-1}<K_{v-2} \ldots \ldots\right.\right.} \\
& {\left[\mathrm{H}^{+}\right] \gg K_{v+1}>K_{v+2} \ldots \ldots}
\end{aligned}
$$

weshalb, wenn $K_{1} \ldots K_{\nu-1}, K_{\nu+1}, K_{\nu+2} \ldots \ldots$ unbekannt sind, zur Berechnung des ersten Näherungswertes von $K_{\nu}$ gesetzt werden darf:

$$
\begin{aligned}
& {\left[\mathrm{A}_{\nu-1}{ }^{-}\right] \sim C_{\nu-1}} \\
& {\left[\mathrm{~A}_{\nu-2}\right] \sim C_{\nu-2}} \\
& \begin{array}{c}
\left.-\overline{A_{v+1}}\right] \sim 0 \\
{\left[\mathrm{~A}_{v+1}\right]}
\end{array} \\
& {\left[\mathrm{A}_{\boldsymbol{v}+2}^{-}\right] \sim 0} \\
& \text { - - - - - }
\end{aligned}
$$

Die obigen Gleichungen sind für ein Säure- bzw. Basen-Gemisch abgeleitet worden. Sie gelten deshalb nicht exakt für mehrbasische Säuren bzw. mehrsäurige Basen, die in einem Molekül mehrere Säure- bzw. Basenfunktionen haben, wie dies betreffs des unhydrolysierten Hämoglobins der Fall ist. Die Gleichungen (1) liefern indessen praktisch genaue Werte in dem Fall, dass $K_{v} / K_{v+1} \geqq 10^{2}$. Beim Hämoglobin liegen die Konstanten näher einander. Aber die Messungsfehler sind in diesem Fall so gross, dass die Gleichungen (1) trotzdem verwendbar sind. Um auf jedem Fall die Grösse des möglichen Fehlers zu prüfen, wurden $K_{1}, K_{2}, K_{3}{ }^{\prime}$ und $K_{3}$ " des menschlichen Hämoglobins ausser mit Hilfe der Gleichungen (1) auch mit Hilfe der für mehrbasische Säuren geltenden weniger übersichtlichen Gleichungen:

$$
\begin{aligned}
& \mathbf{K}_{v}=\left[\mathrm{H}^{+}\right] \frac{\left([\mathrm{B}]+\left[\mathrm{H}^{+}\right]-\left[\mathrm{OH}^{-}\right]\right) \mathbf{M}-\mathbf{N}}{\mathrm{O}-\left([\mathrm{B}]+\left[\mathrm{H}^{+}\right]-\left[\mathrm{OH}^{-}\right]\right) \mathbf{R}} \\
& \mathbf{M}=1+\frac{\left[\mathrm{H}^{+}\right]}{K_{\nu-1}}+\frac{\left[\mathrm{H}^{+}\right]^{2}}{K_{\nu-1} \cdot K_{\nu-2}}+\ldots \ldots+\frac{\left[\mathrm{H}^{+}\right]^{\nu-1}}{K_{\nu-1} \cdot K_{\nu-2} \ldots K_{1}} \\
& \mathrm{~N}=\sum_{i=1}^{\nu-1} C_{i}+\frac{\left[\mathrm{H}^{+}\right]}{K_{\nu-1}} \sum_{i=1}^{\nu-2} C_{i}+\frac{\left[\mathrm{H}^{+}\right]^{2}}{K_{\nu-1} \cdot K_{\nu-2}} \sum_{i=1}^{\nu-3} C_{i}+\ldots+\frac{\left[\mathrm{H}^{+}\right]^{\nu-2}}{K_{\nu-1} \ldots K_{2}} C_{1} \\
& \mathrm{O}=\sum_{i=1}^{\nu} C_{i}+\frac{K_{\nu+1}}{\left[\mathrm{H}^{+}\right]} \sum_{i=1}^{\nu+1} C_{i}+\frac{K_{\nu+1} \cdot K_{v+2}}{\left[\mathrm{H}^{+}\right]^{2}} \sum_{i=1}^{v+2} C_{i}+\ldots+\frac{K_{\nu+1} \cdot K_{v+2} \cdots K_{n}}{\left[\mathrm{H}^{+}\right]^{\mathrm{n}-\nu}} \sum_{i=1}^{\mathrm{n}} C_{i} \\
& \mathbf{R}=1+\frac{K_{v+1}}{\left[\mathrm{H}^{+}\right]}+\frac{K_{\nu+1} \cdot K_{v+2}}{\left[\mathrm{H}^{+}\right]^{2}}+\ldots+\frac{K_{v+1} \cdot K_{v+2} \ldots K_{n}}{\left[\mathrm{H}^{+}\right]^{n-v}}
\end{aligned}
$$


berechnet (vgl. Tabelle 6a). Die Übereinstimmung ist für den vorliegenden Zweck genügend, denn der Unterschied steigt nicht über die Versuchsfehler und hat keinen prinzipiellen Einfluss auf die Resultate. Der verhältnismässig grössere Unterschied bei $K_{4}, K_{5}$ und $K_{6}$ wird verursacht durch die Vergrösserung der Faktoren $\sum_{i=1}^{v-1} C_{i}$ und $\sum_{i=1}^{v+1} C_{i}$; denn die nach (3) zu berechnenden Werte von $K$ werden dadurch stärker von den möglichen Fehlern in $C_{i}$ und von den Fehlern in den naheliegenden Werten von $K$ beeinflusst.

Die Gleichungen (3) wurden durch Verallgemeinerung der für eine vierbasische Säure geltenden Gleichung abgeleitet. Es sei hier die Ableitung der betreffenden Gleichungen für eine zweibasische Säure dargestellt*), weil dieselben zur Berechnung der Dissoziationskonstanten von Hämin verwendet wurden.

Aus den Gleichungen:

$$
\begin{gathered}
\frac{\left[\mathrm{H}^{+}\right]\left[\mathrm{HA}^{-}\right]}{\left[\mathrm{H}_{2} \mathrm{~A}\right]}=K_{1} ; \frac{\left[\mathrm{H}^{+}\right]\left[\mathrm{A}^{=}\right]}{\left[\mathrm{HA}^{-}\right]}=K_{2} \\
{\left[\mathrm{H}_{2} \mathrm{~A}\right]+\left[\mathrm{HA}^{-}\right]+\left[\mathrm{A}^{-}\right]=C ;[\mathrm{B}]+\left[\mathrm{H}^{+}\right]=\left[\mathrm{HA}^{-}\right]+2\left[\mathrm{~A}^{=}\right]+[\mathrm{OH}]}
\end{gathered}
$$

ergeben sich für eine zweibasische Säure die Gleichungen:

$$
\left.\begin{array}{rl}
K_{1} & =\left[\mathrm{H}^{+}\right] \frac{\left([\mathrm{B}]+\left[\mathrm{H}^{+}\right]-\left[\mathrm{OH}^{-}\right]\right)}{\mathrm{C}\left(1+\frac{2 K_{2}}{\left[\mathrm{H}^{+}\right]}\right)-\left([\mathrm{B}]+\left[\mathrm{H}^{+}\right]-\left[\mathrm{OH}^{-}\right]\right)\left(1+\frac{K_{2}}{\left[\mathrm{H}^{+}\right]}\right)} \\
K_{2} & =\left[\mathrm{H}^{+}\right] \frac{\left([\mathrm{B}]+\left[\mathrm{H}^{+}\right]-\left[\mathrm{OH}^{-}\right]\right)\left(1+\frac{\left[\mathrm{H}^{+}\right]}{K_{1}}\right)-C}{2 C-\left([\mathrm{B}]+\left[\mathrm{H}^{+}\right]-[\mathrm{OH}]\right)}
\end{array}\right\}
$$

die sich zur Berechnug von $K_{1}$ und $K_{2}$ durch Annäherungsrechnung eignen. Im vorliegenden Fall sind $\left[\mathrm{H}^{+}\right]$und $\left[\mathrm{OH}^{-}\right]$im Vergleich zu [B] so klein, dass sie neben $[\mathrm{B}]$ unberücksichtigt bleiben können. Sie sind jedoch der Vollständigkeit halber in die Gleichungen mitgenommen worden, weil sie bei sehr starken bzw. sehr schwachen Säuren zu berücksichtigen sind. Bei der Berechnung des ersten Näherungswertes von $K_{1}$ darf $2 K_{2} /\left[\mathrm{H}^{+}\right]$neben 1 vernachlässigt werden und ebenso $\left[\mathrm{H}^{+}\right] / K_{1}$ neben 1 bei der Berechnung des ersten Näherungswertes von $K_{2}$.

* Die früher in anderem Zusammenhang für zweibasische Säuren mitgeteilte Gleichung (Kilpi, S., und Harjanne, P. Suomen Kemistilehti B, 21, 1948, 14-18) ist fehlerhaft; doch wirkt der betreffende Fehler auf das Berechnungsresultat nur ein, wenn $K_{1}$ und $K_{2}$ nahe einander liegen. In dem zitierten Fall ist der Einfluss des fehlerhaften Ausdrucks auf das Berechnungsresultat von untergeordneter Bedeutung. 


\section{DIE DISSOZIATIONSKONSTANTEN VON HÄMOGLOBIN}

Von den Dissoziationskonstanten des Hämoglobins wurden die zwischen den Inflexionspunkten wirkenden zuerst berechnet, weil deren Berechnung verhältnismässig wenig von den übrigen Dissoziationskonstanten abhängig ist. Beim Untersuchen von fabriksmässig hergestelltem Hämoglobin wurde für dieses Intervall nur eine einzige Dissoziationskonstante ermittelt, deren Grösse etwa $10^{-7}$ betrug ${ }^{2}$. Von derselben Grössenordnung ist der sich nach den nachstehend dargestellten Messungen ergebende Mittelwert der im Zwischengebiet der Inflexionspunkte gefundenen Werte der Dissoziationskonstante beim nativen Hämoglobin, wenn vorausgesetzt wird, dass in diesem Bereich nur eine Dissoziationskonstante vorkommt. Die ermittelten Werte der Dissoziationskonstante zeigten jedoch die Neigung, bei zunehmender Wasserstoffionenkonzentration zu wachsen. Beim fabriksmässig hergestellten Hämoglobin konnte dies nicht beobachtet werden wegen der Koagulation des Hämoglobins bei $\mathrm{p}(a \mathrm{H})=7,2$, wobei die Fällung beim Wachsen der Wasserstoffionenkonzentration fortwährend zunahm. Beim nativen Hämoglobin ergab sich die beste Konstanz der Dissoziationskonstante bei Annahme von zwei Dissoziationskonstanten zwischen den Inflexionspunkten und mit der Annahme, dass der Äquivalentpunkt beim "Neutralpunkt» $(\mathrm{p} a \mathrm{H}=7,5)$ liegt, d.h. in der ursprünglichen Lösung, die weder mit Säure noch mit Base versetzt worden war.

Die Werte aller übrigen Dissoziationskonstanten wurden mit Hilfe der Gleichungen (1) durch Annäherungsrechnung ermittelt. Der Endpunkt der Titration auf der sauren Seite, der als "Anfangspunkt" in den Berechnungen verwendet wurde, wurde ermittelt durch Ausprobieren mit Hilfe der Gleichung ${ }^{3}$ :

$$
\left[\mathrm{H}^{+}\right]=\sqrt{\sum_{i=1}^{n} K_{i} C_{i} /\left(1+\frac{K_{i}}{\left[\mathrm{H}^{+}\right]}\right)}
$$

wobei die gemessene $\mathrm{H}^{+}$-Konzentration mit der nach dieser Gleichung berechneten übereinstimmen soll. Die Bestimmung des Endpunktes der Titration auf der alkalischen Seite beim Titrieren mit einer Base geschah in entsprechender Weise mit Hilfe der Gleichung ${ }^{3}$ :

$$
\left[\mathrm{OH}^{-}\right]=\sqrt{\sum_{i=n}^{1} \frac{K_{W}}{K_{i}} C_{i} /\left(1+\frac{\left[\mathrm{H}^{+}\right]}{K_{i}}\right)}
$$

Die Äquivalentpunkte wurden durch Ausprobieren so gewählt, dass der Gang der Dissoziationskonstanten zwischen ihnen, welcher bei unrichtig gewählten Äquivalentpunkten vorkommt, möglichst ausblieb (vgl. Tab. 6). 
In den Berechnungen wurden folgende für 0,1 $\mathrm{N} \mathrm{NaCl-Lösungen} \mathrm{früher}$ ermittelte Beziehungen verwendet:

$$
\begin{gathered}
\mathrm{p}(a \mathrm{H})=\mathrm{p}(\mathrm{cH})+0,085 \\
{\left[\mathrm{H}^{+}\right]\left[\mathrm{OH}^{-}\right]=1,65 \cdot 10^{-145}}
\end{gathered}
$$

Die für Menschen-, Pferde- und Rinderhämoglobin bei $25^{\circ} \mathrm{C}$ in $0,1 \mathrm{~N}$ NaCl-Lösung erhaltenen Werte der Dissoziationskonstanten, die Konzentrationen $C$ der verschiedenen Säure- bzw. Basenfunktionen und deren Äquivalentgewichte $\ddot{A}\left(=\frac{\text { g-Hämogl./ }}{C}\right)$ sowie die Zahl $n$ der verschiedenen Säure- bzw. Basenfunktionen im Hämoglobinmolekül $\left(n=\frac{67000}{\ddot{A}}\right)$ befinden sich in den Tabellen 2-4. In diesen sind weiter die Konzentration des Hämoglobins in der Lösung und die $\mathrm{p}(a \mathrm{H})$-Werte der obengenannten Inflexionspunkte angegeben. Die Werte der Inflexionspunkte wurden in dieser Arbeit mit. Hilfe der von Meretoja hergeleiteten Gleichung ${ }^{13}$ berechnet.

Tabelle 2. Menschliches Hämoglobin, 5,88 g/l. Die Inflexionspunkte bei $p(a H)=5,76$ und 8,70 .

\begin{tabular}{|c|c|c|c|c|c|c|c|}
\hline & $\mathrm{HA}_{1}$ & $\mathrm{HA}_{2}$ & $\mathrm{HA}_{3}^{\prime}$ & $\mathrm{HA}_{3}^{\prime \prime}$ & $\mathrm{HA}_{4}$ & $\mathrm{HA}_{5}$ & $\mathrm{HA}_{6}$ \\
\hline & & & & & & & \\
$\mathrm{C} \cdot 10^{3}$ & 2.95 & 3,81 & 1,676 & 0,884 & 1,1 & 2,743 & 4,86 \\
$\mathrm{p} K$ (Gl. 1) & 3,15 & 4,35 & 6,69 & 7,63 & 9,82 & 10,73 & 11,37 \\
$p K$ (Gl. 3) & 3,09 & 4,32 & 6,65 & 7,61 & 9,63 & 10,56 & 11,45 \\
$A$ & 2000 & 1550 & 3500 & 6650 & 5350 & 2150 & 1210 \\
$\mathrm{n}$ & 33 & 43 & 19 & 10 & 13 & 31 & 55 \\
\hline
\end{tabular}

Tabelle 3. Pferdehämoglobin, $8,28 \mathrm{~g} / \mathrm{l}$. Die Inflexionspunkte bei $p(a H)=5,77$ und 8,79 .

\begin{tabular}{|l|c|c|c|c|c|c|c|}
\hline & $\mathrm{HA}_{1}$ & $\mathrm{HA}_{2}$ & $\mathrm{HA}_{3}^{\prime}$ & $\mathrm{HA}_{3}^{\prime \prime}$ & $\mathrm{HA}_{4}$ & $\mathrm{HA}_{5}$ & $\mathrm{HA}_{6}$ \\
\hline & & & & & & & \\
$\mathrm{C} \cdot 10^{3}$ & 4,23 & 3,5 & 2,1 & 1,4 & 1,5 & 3,75 & 4 \\
$\mathrm{p} K$ & 3,21 & 4,28 & 6,70 & 7,66 & 9,87 & 10,73 & 11,38 \\
$A$ & 1960 & 2370 & 3940 & 5,900 & 5500 & 2210 & 2070 \\
$\mathrm{n}$ & 34 & 28 & 17 & 11 & 12 & 30 & 32 \\
\hline
\end{tabular}

Tabelle 4. Rinderhämoglobin, $8,61 \mathrm{~g} / \mathrm{l}$. Die Inflexionspunkte bei $p(a \mathrm{H})=5,77$ und 8,72.

\begin{tabular}{|l|c|c|c|c|c|c|c|}
\hline & $\mathrm{HA}_{1}$ & $\mathrm{HA}_{2}$ & $\mathrm{HA}_{3}^{\prime}$ & $\mathrm{HA}_{3}^{\prime \prime}$ & $\mathrm{HA}_{4}$ & $\mathrm{HA}_{5}$ & $\mathrm{HA}_{6}$ \\
\hline $\mathrm{C} \cdot 10^{3}$ & 7,95 & 3,715 & 2,0 & 1,0 & 1,6 & 4,15 & 4,25 \\
$\mathrm{p} K$ & 3,46 & 4,23 & 6,71 & 7,71 & 10,04 & 10,66 & 11,45 \\
$A$ & 1080 & 23,20 & 4280 & 8450 & 5380 & 2075 & 2025 \\
$\mathrm{n}$ & 62 & 29 & 16 & 8 & 12,5 & 32 & 33 \\
\hline
\end{tabular}




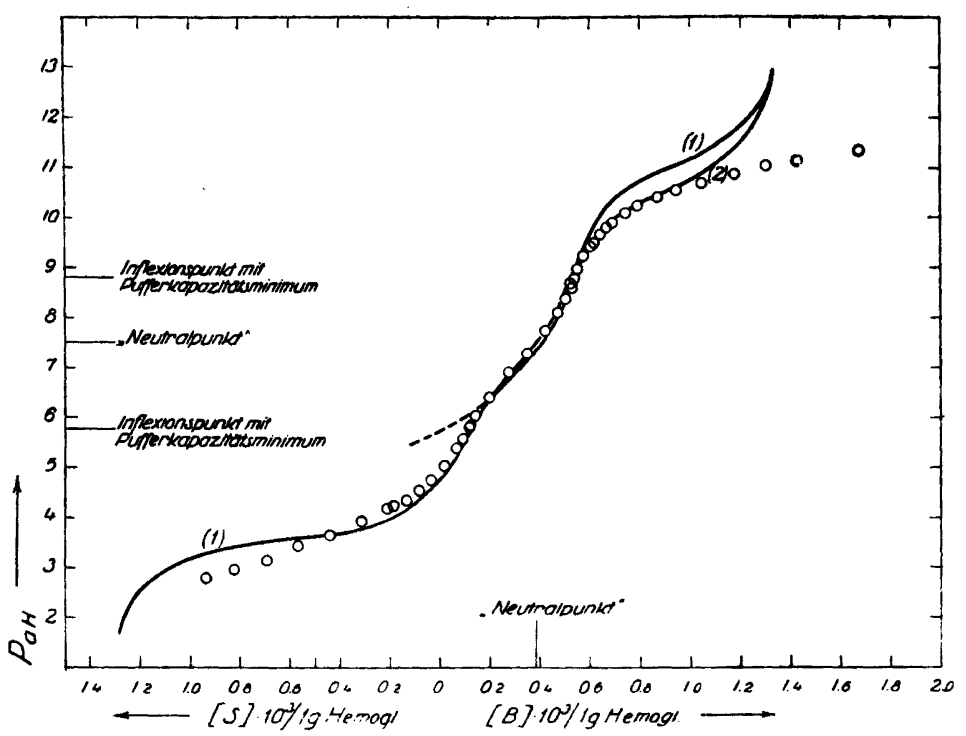

Abbildung 1. Titrierkurven von Pferdehämoglobin.

Kurve (1) nach Cohn und Mitarb. ohne NaCl-Zusatz.

" (2) " " " in $1 / 1 \mathrm{~N} \mathrm{NaCl}$.

○ Titrierungswerte von Kilpi in 0,1 N NaCl; bei diesen ist $[\mathrm{OH}]$ bzw. $\left[\mathrm{H}^{+}\right]$nicht von dem bezüglichen Basenbzw. Säureverbrauch abgezogen.

Den Tabellen gemäss sind die für Menschen-, Pferde- und Rinderhämoglobin erhaltenen, einander entsprechenden Dissoziationskonstanten von der gleichen Grössenordnug. Zwischen den Inflexionspunkten ist nach den Tabellen die Zahl der Säure-Basen-Funktionen in einem Hämoglobinmolekül am kleinsten beim Rinderhämoglobin. Damit hängt zusammen der aus Abb. 2 ersichtliche, im Vergleich zu den anderen Titrationskurven grössere Anstieg der Titrationskurve des Rinderhämoglobins zwischen den Inflexionspunkten, wonach die Pufferkapazität des Rinderhämoglobins zwischen den Inflexionspunkten etwas kleiner ist als die Pufferkapazität des Menschen- und Pferdehämoglobins.

Abb. 1 zeigt, wie sich die gemessenen $\mathrm{p}(a \mathrm{H})$-Werte beim Titrieren von Pferdehämoglobin der von Cohn, Green und Blanchard gegebenen Titrationskurve von Pferdehämoglobin (CO-Hämoglobin) ${ }^{6}$ anpassen. Als 0-Punkt der Titration ist in der Abbildung der von Cohn und Mitarb. verwendete angenommen. Obgleich die Hämoglobinlösungen in ganz verschiedener Weise bereitet worden sind, ist die Ubereinstimmung innerhalb des $\mathrm{p}(a \mathrm{H})$-Bereichs 


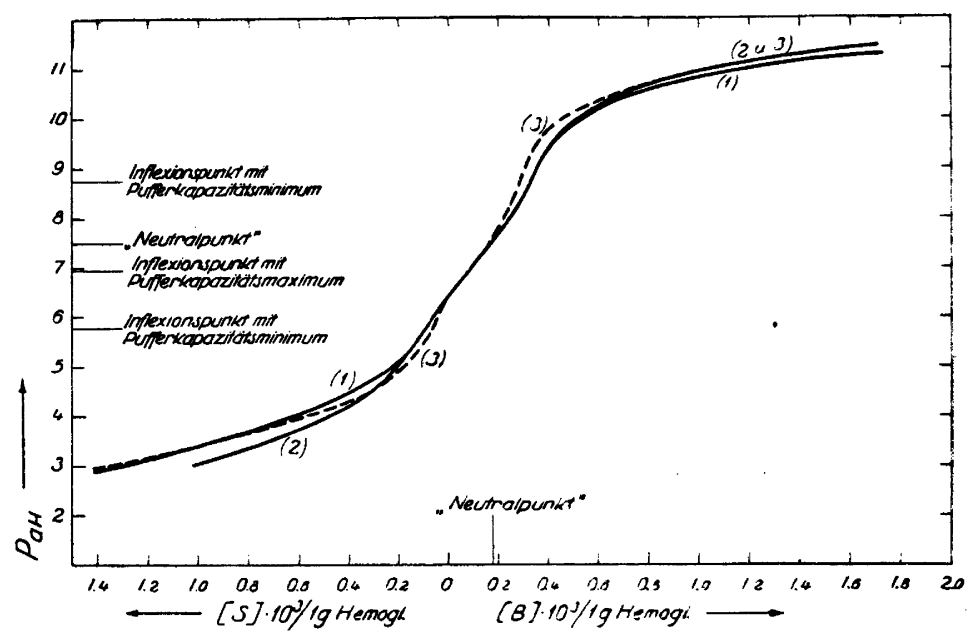

Abbildung 2. Titrierkurven von Menschen (1)-, Pferde (2)- und Rinderhämoglobin (3) in 0,1 N NaCl.

4-9,5 gut, am besten in dem physiologisch wichtigsten Bereich zwischen den Inflexionspunkten. Die in den stärker sauren bzw. stärker basischen Lösungen eintretende Abweichung unserer Werte von der Cohn'schen Titrationskurve dürfte weningstens zum Teil durch die ungleiche Hydrolyse des Hämoglobins verursacht sein.

Während unsere Titrationskurve in ihrem wichtigsten Teil mit der von Cohn und Mitarb. übereinstimmt, weichen die erhaltenen Konstanten mehr voneinander ab. Cohn und Mitarb. teilen folgende Werte für die Säure-Dissoziationskonstanten des Hämoglobins mit:

$$
\begin{array}{lllllll}
\mathrm{p} K_{1}, & \mathrm{p} K_{2}, & \mathrm{p} K_{3}, & \mathrm{p} K_{4}, & \mathrm{p} K_{5}, & \mathrm{p} K_{6}, & \mathrm{p} K_{7} \\
3,7 & 4,0 & 4,8 & 5,7 & 7,5 & 10,8 & 11,6
\end{array}
$$

Es möge bemerkt werden, dass beim Berechnen von $\mathrm{p}(a \mathrm{H})$ mit Hilfe der Gleichungen (1) und (7) sich Werte ergeben, die sich mit den Titrationskurven in Abb. 2 decken, sofern man sich bei den Berechnungen der in den Tabellen 2-4 angegebenen Dissoziationskonstanten und Konzentrationen bedient.

\section{DIE PUFFERWIRKUNG DES BLUTES}

Sind die Konzentrationen und die Dissoziationskonstanten der in der Lösung befindlichen Säuren bzw. Basen bekannt, kann die Pufferkapazität 
bei verschiedenen $\mathrm{H}^{+}$-Konzentrationen mit Hilfe der van Slyke'schen ${ }^{7}$ Gleichung:

$$
\frac{\mathrm{d}[\mathrm{B}]}{\mathrm{d} \mathrm{pH}}=P=2,303\left\{\sum_{i} \frac{K_{i} C_{i}\left[\mathrm{H}^{+}\right]}{\left(K_{i}+\left[\mathrm{H}^{+}\right]\right)^{2}}+\left[\mathrm{H}^{+}\right]+\left[\mathrm{OH}^{-}\right]\right\}
$$

berechnet werden, wo $K_{i}$ die Konzentrationsdissoziationskonstanten der Säuren bzw. die Hydrolysekonstanten ${ }^{3}$ der Basen-Kationen sind.

Mit Hilfe dieser Gleichung wurde die Pufferkapazität des Blutes bei $\mathrm{p}(a \mathrm{H})$ $=7,3$ und 6,5 berechnet, unter der Annahme, dass die Pufferkapazität vom Hämoglobin sowie von Kohlensäure und Phosphorsäure des Blutes herrührt. Die Pufferwirkung des Hämoglobins bei $\mathrm{p}(a \mathrm{H})=7,3$ and 6,5 ist hauptsächlich von den zwischen den Inflexionspunkten wirkenden Dissoziationskonstanten $K_{3}^{\prime}$ und $K_{3}^{\prime \prime}$ abhängig, während die Pufferwirkung der Kohlensäure bei diesen $\mathrm{p}(a \mathrm{H})$-Werten hauptsächlich von der ersten Dissoziationskonstante und die der Phosphorsäure hauptsächlich von der zweiten abhängig ist. Betreffs der Kohlensäure wurde die zweite Dissoziation gleichfalls berücksichtigt, obgleich deren Wirkung von untergeordneter Bedeutung ist, weshalb zur Berechnung der betreffenden Pufferwirkung ein angenäherter Wert dieser Dissoziationskonstante genügt.

Die betreffenden Konzentrationsdissoziationskonstanten wurden berechnet für die Ionenstärke $I=0,1 \mathrm{mit} \mathrm{NaCl}$ als hauptsächlicher Elektrolyt, weil die Ionenstärke des Blutes annähernd von dieser Grösse ist. Die nach der DebyeHückelschen Theorie berechneten Konzentrationsdissoziationskonstanten der Kohlensäure ${ }^{8}$ und der Phosphorsäure ${ }^{9}$ und die stöchiometrischen molaren Konzentrationen $C$ derselben im Blut sind:

\begin{tabular}{|c|c|c|c|}
\hline & $K_{1}$ & $K_{2}$ & $C$ \\
\hline Kohlensäure & $7,76 \cdot 10^{-7}$ & $10^{-10}$ & 0,0245 \\
Phosphorsäure & & $1,8 \cdot 10^{-7}$ & 0,0013 \\
\hline
\end{tabular}

Bei der Berechnung der Pufferkapazität des Hämoglobins wurde das Blut als $16 \%$ ig in bezug auf das Hämoglobin angenommen. Man erhält dann die Konzentrationen der verschiedenen Säurefunktionen des Hämoglobins im Blut durch Multiplizieren der in den Tabellen 2-4 angegebenen Konzentrationen $C$ beim menschlichen Hämoglobin mit 16/0,584, beim Pferdehämoglobin mit 16/0,828 und beim Rinderhämoglobin mit 16/0,861. Die Rechnung ergab für die Pufferkapazität bei $\mathrm{p}(a \mathrm{H})=7,3$ und 6,5 die Werte der Tabelle 5 . 
Tabelle 5. Pufferkapazität (P).

\begin{tabular}{|l|l|r|r|r|r|r|}
\hline & \multicolumn{3}{|r|}{ In Menschenblut } & \multicolumn{2}{|r|}{ In Pferdeblut } & \multicolumn{2}{|c|}{ In Rinderblut } \\
& $p_{\mathrm{aH}}=7,3$ & $\mathrm{p}_{\mathrm{aH}}=6,5$ & $\mathrm{p}_{\mathrm{aH}}=7,3$ & $\mathrm{p}_{\mathrm{aH}}=6,5$ & $\mathrm{p}_{\mathrm{aH}}=7,3$ & $\mathrm{p}_{\mathrm{aH}}=6,5$ \\
\hline & & & & & & \\
Hämoglobin & $3,04 \cdot 10^{-2}$ & $2,85 \cdot 10^{-2}$ & $2,90 \cdot 10^{-2}$ & $2,60 \cdot 10^{-2}$ & $2,37 \cdot 10^{-2}$ & $2,22 \cdot 10^{-2}$ \\
Kohlensäure & $0,48 \cdot 10^{-2}$ & $1,27 \cdot 10^{-2}$ & $0,48 \cdot 10^{-2}$ & $1,27 \cdot 10^{-2}$ & $0,48 \cdot 10^{-2}$ & $1,27 \cdot 10^{-2}$ \\
Phosphorsäure & $0,06 \cdot 10^{-2}$ & $0,06 \cdot 10^{-2}$ & $0,06 \cdot 10^{-2}$ & $0,06 \cdot 10^{-2}$ & $0,06 \cdot 10^{-2}$ & $0,06 \cdot 10^{-2}$ \\
\hline $\begin{array}{l}P \text { des Blutes: } \\
\text { berechnet } \\
\text { gemessen }\end{array}$ & $3,58 \cdot 10^{-2}$ & $4,18 \cdot 10^{-2}$ & $3,44 \cdot 10^{-2}$ & $3,93 \cdot 10^{-2}$ & $2,91 \cdot 10^{-2}$ & $3,55 \cdot 10^{-2}$ \\
\cline { 2 - 6 } & $3,61 \cdot 10^{-2}$ & $4,25 \cdot 10^{-2}$ & $3,26 \cdot 10^{-2}$ & $4,00 \cdot 10^{-2}$ & $2,97 \cdot 10^{-2}$ & $3,59 \cdot 10^{-2}$ \\
\hline
\end{tabular}

Gemäss der Tabelle 5 wird die Pufferkapazität des Blutes hauptsächlich durch das Hämoglobin verursacht. Der Anteil der Kohlensäure an der Pufferwirkung variiert jedoch stark mit der Veränderung der $\mathbf{H}^{+}$-Konzentration der Lösung.

Zum Vergleich mit den mit Hilfe der erhaltenen Dissoziationskonstanten berechneten Werten wurde die Pufferkapazität des Blutes direkt durch Titration gemessen. Dazu wurden im Verhältnis von $1 / 10$ verdünnte und in bezug auf $\mathrm{NaCl}$ 0,1-normale Blutlösungen verwendet, nachdem durch Titration gezeigt worden war, dass sich die Pufferkapazität des Blutes innerhalb dieses Intervalls linear mit der Konzentration verändert.

Das $\mathrm{p}(a \mathrm{H})$ des «Neutralpunktes» des Blutes, zu dem keine Säure bzw. Base zugesetzt worden war, war 7,5 \pm 0,1, wie beim »Neutralpunkt» des Hämoglobins (vgl. Abb. 1 und 2). Auch hat die Titrationskurve des Blutes (vgl. Abb. 3 und die Tabellen 8-10) der Titrationskurve des Hämoglobins entsprechend zwei Inflexionspunkte mit Pufferkapazitätsminima, deren $\mathrm{p}(a \mathrm{H})$ Werte annähernd gleich den $\mathrm{p}(a \mathrm{H})$-Werten der Inflexionspunkte des Hämoglobins sind.

In der Tabelle 5 sind die berechneten Pufferkapazitätswerte bei $\mathrm{p}(a \mathrm{H})=$ 7,3 und bei $\mathrm{p}(a \mathrm{H})=6,5$ den sich nach den Titrationskurven ergebenden Werten nebengestellt. Die berechneten und gemessenen Werte der Pufferkapazität zeigen eine gute Übereinstimmung. Die Titrationskurven des Menschen- und Rinderblutes waren gut.reproduzierbar. Beim Pferdeblut war die Reproduzierbarkeit schlechter, wahrscheinlich wegen der Neigung zur Sedimentation. In bezug auf das Rinderblut wurde erwiesen, dass der Gang der Titrationskurve von dem Alter des Tieres unabhängig ist. Die aus der Abb. 3 und nach der Tabelle 5 ersichtliche geringe gegenseitige Abweichung der Pufferkapazitätswerte des Menschen-, Pferde- und Rinderblutes voneinander (Menschenblut $>$ Pferdeblut $>$ Rinderblut) dürfte deshalb durch die Titrationen mit ziemlich 


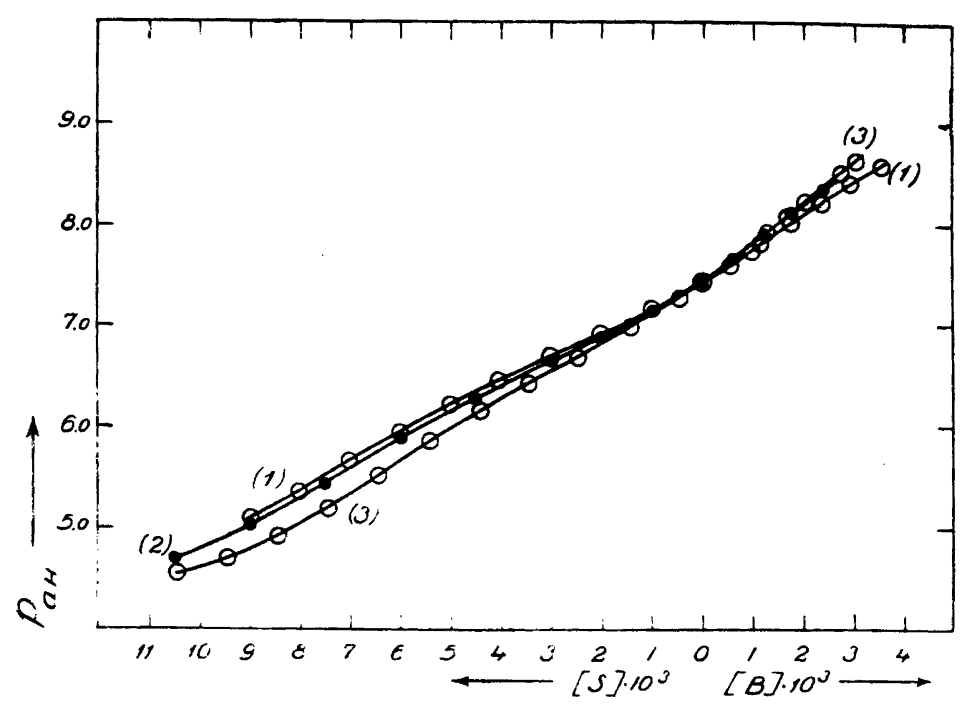

Abbildung 3. Titrierkurven von in Verhältnis von $1 / 10$ verdünnten Menschen (1)-, Pferde (2)- und Rinderblutlösungen (3) in 0,1 N NaCl.

grosser Sicherheit erwiesen sein. Diese Abweichung würde durch den verschiedenen Gehalt des Hämoglobins an Säure-Basenfunktionen mit den Dissoziationskonstanten $K_{3}{ }^{\prime}$ und $K_{3}{ }^{\prime \prime}$ verursacht. Die Grössen dieser Konstanten waren nämlich gemäss den Messungen gleich, unabhängig von der Herkunft des Hämoglobins.

\section{DIE DISSOZIATIONSKONSTANTEN VON HÄMATIN IM VERGLEICH MIT DENEN DES HÄMOGLOBINS}

Weil der Hämatingehalt des Hämoglobins ziemlich gross ist (vier Hämatingruppen pro Hämoglobinmolekül ${ }^{10}$ ), war es von Interesse, die SäureBasen-Dissoziationskonstanten des Hämatins mit denen des Hämoglobins zu vergleichen.

Bei der Bestimmung der Dissoziationskonstanten von Hämatin wurde zuerst ein Hämatin-Präparat von Fraenkel \& Landau verwendet ${ }^{2}$. Dieses löste sich doch nur teilweise in Natriumhydroxyd. Die folgenden Messungsresultate sind mit in Natriumhydroxyd gelöstem Hämin (krist. reinst, Fraenkel \& Landau) erhalten, wobei die Titrationskurve wie bei Anwendug von Hämatin zwei Inflexionspunkte hatte. 
Für die Dissoziationskonstanten konnten nur angenäherte Werte erhalten werden. Die Unsicherheit der Messungen beruht hauptsächlich darauf, dass wegen der Schwerlöslichkeit des Hämins nur äusserst verdünnte Lösungen $(0,68$ $\mathrm{g} / \mathrm{l}$ ) verwendet werden konnten.

Den Hämoglobinlösungen entsprechend hatte die Titrierungskurve des Hämins zwei deutliche Inflexionspunkte, nämlich bei $\mathrm{p}(a \mathrm{H})=5,51$ und 9,13 bei obiger Konzentration der Lösung. Beim sauren Inflexionspunkt fiel das Hämin aus. Die stöchiometrische Konzentration der Basenmenge, die bei der Titrierung von dem ersten Inflexionspunkt zum anderen zuzusetzen war, betrug $2 \cdot 10^{-3}$. Daraus berechnet sich für das Äquivalentgewicht der Hämins $0,68 / 2 \cdot 10^{-3}=340$, welcher Wert innerhalb der Titrationsfehler mit dem halben Molekülgewicht des Hämins 325,8 übereinstimmt. Dieses ist damit in Übereinstimmung, dass das Hämin zwei Caboxylgruppen enthält. Für die $\mathrm{p} K_{A}$-Werte der Dissoziationskonstanten wurde $6,63 \mathrm{bzw} .7,76$ erhalten, welche Werte von derselben Grössenordnung sind wie die erhaltenen $\mathrm{p} K_{3}^{\prime}$ und $\mathrm{p} K_{3}^{\prime \prime}$ des Hämoglobins.

Unter der Annahme, dass die Konstanten des Hämins gleich denen des Hämatins sind, ist deshalb die Pufferwirkung des Hämoglobins zwischen den Inflexionspunkten von dem Hämatingehalt des Blutes abhängig, wie auch auf Grund der Titration von Hämatin zu schliessen war ${ }^{2}$. Weil ein Hämoglobinmolekül vier Hämatingruppen enthält ${ }^{10}$, ist nach unseren Titrationsresultaten (vgl. Tabellen 2-4) zwischen den Inflexionspunkten der Anteil des Hämatins ca. 4/10 der gesamten Pufferwirkung des Hämoglobins. Die Pufferwirkung zwischen den Inflexionspunkten dürfte daneben von Histidylhistidin verursacht sein. Denn das Histidylhistidin hat nach Greenstein ${ }^{11}$ vier Dissoziationskonstanten, von denen zwei mit den $\mathrm{p} K_{A}$-Werten $6,80 \mathrm{bzw}$. 7,80 von ähnlicher Grössenordnung sind.

\section{DIE MESSUNGEN}

$50 \mathrm{ml}$ der betreffenden Hämoglobin-, bzw. Häminlösung wurden mit 0,1 $N$ $\mathrm{NaOH}-$ bzw. 0,1 $N$ HCl-Lösung titriert.

Die $\mathrm{p}(a \mathrm{H})$-Werte der Hämoglobin- und Häminlösungen wurden bestimmt durch Messen des Potentials der Kette:

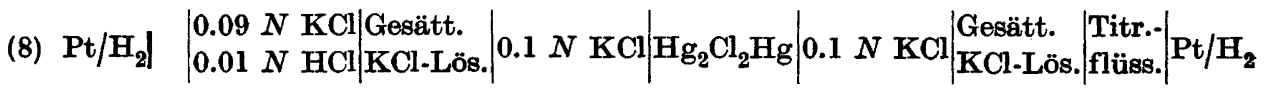

und unter Anwendung von $\mathrm{p}(a \mathrm{H})=2,075$ für die Veibelsche Lösung $(0,09 \mathrm{~N}$ $\mathrm{KCl}+0,01 \mathrm{~N} \mathrm{HCl})$.

Beim Titrieren von Blut wurden die $\mathrm{p}(a \mathrm{H})$-Werte mit einer Glaselektrode gemessen. Die Kettenanordnung war dieselbe wie in (8), jedoch wurde statt 
der Veibelschen Lösung ein Acetat-Puffer mit $\mathrm{p}(a \mathrm{H})=4,65$ als Vergleichslösung ${ }^{12}$ verwendet.

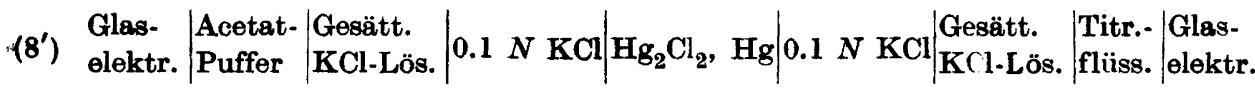

Beim Pferdehämoglobin wurde die Ausführung der Messungen in sauren Lösungen durch die Bildung einer Fällung bei $\mathrm{p}(a \mathrm{H})=4,2$ erschwert. Die

Tabelle 6a. Menschen-Hämoglobin, $5,88 \mathrm{~g} / \mathrm{l}$.

(Die Titrationen sind von A. A. O. Kuosmanen ausgeführt.)

\begin{tabular}{|c|c|c|c|c|c|c|c|}
\hline $\begin{array}{c}E \\
\mathbf{m V}\end{array}$ & $\mathrm{p}(a \mathrm{H})$ & {$\left[\mathrm{H}^{+}\right]$} & {$[\mathrm{OH}]$} & {$[B] \cdot 10^{3}$} & $\begin{array}{c}V \\
\mathrm{ml}\end{array}$ & Gl. (1) & $\begin{array}{l}\mathrm{pK} \\
\text { Gl. (3) }\end{array}$ \\
\hline 57,0 & 3,040 & $\begin{array}{r}\text { (ber.) } 1,15 \cdot 10^{-3} \\
1,12 \cdot 10^{-3}\end{array}$ & & 0 & 55,40 & & \\
\hline 58,9 & 3,172 & $8,23 \cdot 10^{-4}$ & & 0,440 & 54,40 & $\mathbf{3 , 3 0}$ & 3,24 \\
\hline 68,4 & 3,232 & $7,16 \cdot 10^{-4}$ & & 0,835 & 55,80 & 3,20 & 3,15 \\
\hline 71,0 & 3,276 & $6,47 \cdot 10^{-4}$ & & 1,277 & $\mathbf{5 4 , 8 0}$ & 3,05 & 3,00 \\
\hline 81,0 & 3,446 & $4,38 \cdot 10^{-4}$ & & 1,673 & 56,15 & 3,17 & 3,12 \\
\hline 84,6 & 3,507 & $3,80 \cdot 10^{-4}$ & & 2,110 & 55,15 & 3,04 & 2,98 \\
\hline 94,6 & 3,676 & $2,58 \cdot 10^{-4}$ & & 2,507 & 56,52 & 3,14 & 3,06 \\
\hline Äquiv. & & & & & & & \\
\hline 99,1 & 3,752 & $2,16 \cdot 10^{-4}$ & & 2,949 & 55,50 & 3,15 & 3,09 im Mittel \\
\hline 112,6 & 3,980 & $1,28 \cdot 10^{-4}$ & & 3,343 & 56,90 & 4,35 & 4,34 \\
\hline 115,2 & 4,024 & $8,65 \cdot 10^{-5}$ & & 3,783 & 55,92 & 4,38 & 4,38 \\
\hline 131,5 & 4,300 & $6,12 \cdot 10^{-5}$ & & 4,180 & 57,32 & 4,39 & 4,39 \\
\hline 136,9 & 4,391 & $4,97 \cdot 10^{-5}$ & & 4,629 & 56,45 & 4,30 & 4,31 \\
\hline 151,5 & 4,640 & $2,80 \cdot 10^{-5}$ & & 5,020 & 57,78 & 4,41 & 4,43 \\
\hline 152,7 & 4,659 & $2,68 \cdot 10^{-5}$ & & 5,466 & 56,85 & 4,23 & 4,26 \\
\hline 188,2 & 5,259 & $6,73 \cdot 10^{-6}$ & & 6,310 & 57,30 & 4,39 & 4,37 \\
\hline 197,2 & 5,412 & $4,73 \cdot 10^{-6}$ & & 6,520 & 57,40 & 4,38 & 4,28 \\
\hline 207,5 & 5,586 & $3,17 \cdot 10^{-6}$ & & 6,720 & 57,45 & 4,34 & 4,10 \\
\hline 214,6 & 5,706 & $2,33 \cdot 10^{-6}$ & & 6,730 & 54,88 & 4,35 & 4,32 im Mittel \\
\hline Äquiv. & 5,762 & & & 6,755 & 54,80 & & \\
\hline 231,8 & 5,997 & $1,19 \cdot 10^{-6}$ & & 6,932 & 54,72 & 6,69 & 6,66 \\
\hline 247,3 & 6,259 & $6,53 \cdot 10^{-7}$ & & 7,166 & 54,68 & 6,67 & 6,65 \\
\hline 260,8 & 6,488 & $3,86 \cdot 10^{-7}$ & & 7,378 & 54,60 & 6,69 & 6,66 \\
\hline 286,3 & 6,922 & $1,42 \cdot 10^{-7}$ & & 7,790 & 54,32 & 6,77 & 6,78 \\
\hline 312,0 & 7,354 & $5,25 \cdot 10^{-8}$ & & 8,420 & 54,00 & 6,61 & 6,69 \\
\hline Äquiv. & & & & & & 6,69 & 6,65 im Mittel \\
\hline 358,5 & 8,141 & $8,57 \cdot 10^{-9}$ & $2 \cdot 10^{-6}$ & 9,050 & 53,72 & 7,63 & 7,67 \\
\hline 382,5 & 8,547 & $3,39 \cdot 10^{-9}$ & $4 \cdot 10^{-6}$ & 9,272 & 53,70 & 7,62 & 7,55 \\
\hline Äquiv. & 8,698 & & & 9,320 & & 7,63 & 7,61 im Mittel \\
\hline
\end{tabular}


Tabelle 6b. Menschen-Hämoglobin $5,88 \mathrm{~g} / \mathrm{l}$.

\begin{tabular}{|c|c|c|c|c|c|c|}
\hline $\begin{array}{c}E \\
\mathrm{mV}\end{array}$ & $\mathrm{p}(a \mathrm{H})$ & {$\left[\mathrm{H}^{+}\right]$} & {$[\mathrm{OH}]$} & {$[\mathrm{B}] \cdot 10^{3}$} & $\begin{array}{c}V \\
\mathrm{ml}\end{array}$ & $\mathrm{pK}$ (Gl. 1) \\
\hline 420,9 & 9,333 & $5,68 \cdot 10^{-10}$ & $2,9 \cdot 10^{-5}$ & 9,656 & 50,50 & 9,88 \\
\hline 439,3 & 9,508 & $3,80 \cdot 10^{-10}$ & $4,3 \cdot 10^{-5}$ & 9,878 & 50,60 & 9,76 \\
\hline 449,6 & 9,682 & $2,54 \cdot 10^{-10}$ & $6,5 \cdot 10^{-5}$ & 10,070 & 50,65 & 9,79 \\
\hline 459,4 & 9,848 & $1,73 \cdot 10^{-10}$ & $9,5 \cdot 10^{-5}$ & 10,205 & 50,80 & 9,83 \\
\hline Äquiv. & & & & & & 9,82 im Mittel \\
\hline 466,0 & 9,960 & $1,34 \cdot 10^{-10}$ & $1,23 \cdot 10^{-4}$ & 10,504 & 50,90 & 10,75 \\
\hline 477,2 & 10,149 & $8,67 \cdot 10^{-11}$ & $1,90 \cdot 10^{-4}$ & 10,911 & 51,10 & 10,75 \\
\hline 486,6 & 10,309 & $6,00 \cdot 10^{-11}$ & $2,75 \cdot 10^{-4}$ & 11,346 & 51,26 & 10,75 \\
\hline 493,5 & 10,425 & $4,59 \cdot 10^{-11}$ & $3,59 \cdot 10^{-4}$ & 11,767 & 51,48 & 10,71 \\
\hline 499,6 & 10,529 & $3,61 \cdot 10^{-11}$ & $4,57 \cdot 10^{-4}$ & 12,188 & 51,70 & 10,69 \\
\hline 508,3 & 10,676 & $2,58 \cdot 10^{-11}$ & $6,41 \cdot 10^{-4}$ & 12,784 & 51,95 & 10,72 \\
\hline Äquiv. & & & & & & 10,73 im Mittel \\
\hline 521,2 & 10,894 & $1,56 \cdot 10^{-11}$ & $1,058 \cdot 10^{-3}$ & 13,841 & 52,48 & 11,42 \\
\hline 529,3 & 11,031 & $1,14 \cdot 10^{-11}$ & $1,450 \cdot 10^{-3}$ & 14,867 & 52,92 & 11,37 \\
\hline 536,1 & 11,146 & $8,73 \cdot 10^{-12}$ & $1,890 \cdot 10^{-3}$ & 15,957 & 53,55 & 11,32 \\
\hline 547,3 & 11,336 & $5,64 \cdot 10^{-12}$ & $\left\{\begin{array}{l}2,94 \cdot 10^{-3} \\
3,04 \cdot 10^{-3} \text { (ber.) }\end{array}\right.$ & 18,023 & 54,50 & 11,37 im Mittel \\
\hline
\end{tabular}

Fällung bildete sich an dieser Stelle sowohl beim Titrieren mit Chlorwasserstoffsäure als mit Natriumhydroxyd. Im letzteren Falle war zu der Lösung zuerst Chlorwasserstoffsäure in einem solchen Überschuss zugefügt worden, dass die Fällung ausblieb. Beim Menschen- und Rinderblut verblieb die Lösung während der ganzen Titration klar sowohl in sauren als in alkalischen Lösungen.

In den folgenden Tabellen bezeichnet $E$ das Potential der Kette (8) bzw. $\left(8^{\prime}\right)$ und $V$ das Volumen der Lösung in $\mathrm{ml}$. Die in den Tabellen 2-4 angegebenen Konzentrationen $C_{1}, C_{2}, C_{3}{ }^{\prime}, C_{3}{ }^{\prime \prime}, C_{4}, C_{5}$, und $\mathrm{C}_{6}$ sowie die stöchiometrische Konzentration [B] der vom Beginn der Titration an zugesetzten Basenmenge sind auf das ursprüngliche Volumen der Lösung, nämlich $50 \mathrm{ml}$, bezogen. In den Berechnungen ist die tatsächliche Konzentration während der Titration, $C \frac{50}{V}$, verwendet worden. [S] der Tabellen bezeichnet die Konzentration der zugesetzten Säure, weshalb $[\mathrm{B}]=-[\mathrm{S}]$.

Von den ausgeführten Titrationen des Menschen-, Pferde- und Rinderhämoglobins ist hier als Beispiel nur die des menschlichen Hämoglobins vorgelegt worden. Ausserdem sind im folgenden die Titrationen von Hämin sowie die Titrationen des Menschen-, Pferde- und Rinderblutes wiedergegeben.

Alle Titrationen sind bei $25,0^{\circ} \mathrm{C}$ ausgeführt worden. Die Lösungen waren in bezug auf $\mathrm{NaCl}$ 0,1-normal. 
Tabelle .7. Hämin, 0,680 g/l in 0,1 N NaCl-Lösung.

(Die Titrationen sind von Elsa Keränen ausgeführt.)

\begin{tabular}{|c|c|c|c|c|c|c|c|}
\hline $\begin{array}{c}E \\
\mathbf{m V}\end{array}$ & $\mathrm{p}(a \mathrm{H})$ & {$\left[\mathrm{H}^{+}\right]$} & {$[\mathrm{OH}]$} & [B] $10^{3}$ & $\begin{array}{c}V \\
\mathrm{ml}\end{array}$ & $\begin{array}{l}\mathrm{pK} \\
\text { Gl. (1) }\end{array}$ & $\begin{array}{l}\mathrm{pK} \\
\text { Gl. (4) }\end{array}$ \\
\hline 165,6 & 4,875 & $1,62 \cdot 10^{-5}$ & & & 52,80 & & \\
\hline Äquiv. & 5,51 & & & 0 & 52,66 & & \\
\hline 252,5 & 6,347 & $5,50 \cdot 10^{-7}$ & & 0,340 & 52,50 & 6,61 & 6,58 \\
\hline 303,5 & 7,207 & $7,59 \cdot 10^{-8}$ & & 0,934 & 52,20 & 6,64 & 6,61 \\
\hline Äquiv. & & & & 1,000 & & 6,63 & 6,60 im Mittel \\
\hline 328,0 & 7,625 & $2,88 \cdot 10^{-8}$ & & 1,260 & 52,22 & 7,78 & 7,80 \\
\hline 360,9 & 8,180 & $8,04 \cdot 10^{-9}$ & $2 \cdot 10^{-6}$ & 1,661 & 52,02 & 7,74 & 7,77 \\
\hline Äquiv. & 9,13 & & & 2,000 & 51,85 & 7,76 & 7,79 im Mittel \\
\hline 426,5 & 9,285 & $6,31 \cdot 10^{-10}$ & $2,7 \cdot 10^{-5}$ & & 51,82 & & \\
\hline
\end{tabular}

Tabelle 8. Menschenblut.

Die Titrationen von Blut wurden mit Anwendung der Kette (8') ausgeführt. Die Blutlösungen waren im Verhältnis von $1 / 10$ verdünnt und in bezug auf $\mathrm{NaCl} \mathrm{0,1-normal.}$

(Die Titrationen sind von P. J. Väänänen ausgeführt.)

\begin{tabular}{|c|r|r|r|}
\hline$E$ & $\mathrm{p}(a \mathrm{H})$ & {$[\mathrm{S}] \cdot 10^{3}$} & $V(\mathrm{ml})$ \\
\hline 234,75 & 8,62 & $-3,55$ & \\
223,90 & 8,44 & $-2,96$ & 206 \\
212,50 & 8,24 & $-2,37$ & 205 \\
199,65 & 8,03 & $-1,78$ & 204 \\
187,85 & 7,83 & $-1,18$ & 203 \\
176,35 & 7,63 & $-0,59$ & 202 \\
166,90 & 7,47 & 0,00 & 200 \\
150,10 & 7,19 & 1,00 & 202 \\
135,35 & 6,94 & 2,01 & 204 \\
121,85 & 6,71 & 3,01 & 206 \\
108,50 & 6,48 & 4,01 & 208 \\
94,05 & 6,24 & 5,01 & 210 \\
77,30 & 5,96 & 6,01 & 212 \\
60,45 & 5,67 & 7,01 & 214 \\
43,10 & 5,38 & 8,01 & 216 \\
26,15 & 5,09 & 9,01 & 218 \\
\hline
\end{tabular}

Die Inflexionspunkte bei $\mathrm{p}(a \mathrm{H})=5,51$ und 8,11 . 
Tabelle 9. Pferdeblut.

\begin{tabular}{|c|c|r|c|}
\hline$E$ & $\mathrm{p}(a \mathrm{H})$ & {$[S] \cdot 10^{3}$} & $V(\mathrm{ml})$ \\
\hline 219,10 & & & \\
206,10 & 8,35 & $-2,37$ & 204 \\
192,10 & 8,13 & $-1,78$ & 203 \\
180,00 & 7,90 & $-1,18$ & 202 \\
167,80 & 7,69 & $-0,59$ & 201 \\
148,45 & 7,49 & 0,00 & 200 \\
132,05 & 7,16 & 1,00 & 202 \\
120,10 & 6,88 & 2,00 & 204 \\
98,35 & 6,68 & 3,01 & 206 \\
75,25 & 6,31 & 4,52 & 209 \\
48,15 & 5,92 & 6,02 & 212 \\
22,70 & 5,46 & 7,52 & 215 \\
3,30 & 5,03 & 9,02 & 218 \\
\hline
\end{tabular}

Die Inflexionspunkte bei $\mathrm{p}(a \mathrm{H})=5,78$ und 8,06 .

Tabelle 10. Rinderblut.

\begin{tabular}{|c|c|c|c|}
\hline$E$ & $\mathrm{p}(a \mathrm{H})$ & {$[S] \cdot 10^{3}$} & $V(\mathrm{ml})$ \\
\hline 238,10 & 8,68 & $-3,07$ & 204,2 \\
\hline 230,90 & 8,55 & $-2,72$ & 203,6 \\
\hline 222,30 & 8,41 & $-2,36$ & 203,0 \\
\hline 214,70 & 8,28 & $-2,01$ & 202,4 \\
\hline 204,55 & 8,11 & $-1,65$ & 201,8 \\
\hline 194,55 & 7,94 & $-1,30$ & 201,2 \\
\hline 184,75 & 7,77 & $-0,94$ & 200,6 \\
\hline 177,55 & 7,65 & $-0,59$ & 200 \\
\hline 156,10 & 7,29 & $+0,41$ & 202 \\
\hline 140,35 & 7,02 & $+1,42$ & 204 \\
\hline 120,05 & 6,68 & 2,42 & 206 \\
\hline 106,55 & 6,45 & 3,42 & 208 \\
\hline 90,80 & 6,19 & 4,42 & 210 \\
\hline 72,65 & 5,88 & 5,42 & 212 \\
\hline 53,40 & $\mathbf{5 , 5 5}$ & 6,43 & 214 \\
\hline 32,45 & 5,20 & 7,43 & 216 \\
\hline 16,75 & 4,93 & 8,43 & 218 \\
\hline $\mathbf{3 , 9 5}$ & 4,72 & 9,43 & 220 \\
\hline$-6,10$ & 4,55 & 10,43 & 222 \\
\hline
\end{tabular}

Die Inflexionspunkte bei $\mathrm{p}(\mathrm{aH})=5,48$ und 8,11 . 


\section{ZUSAMMENFASSUNG}

Es wurden die Säure-Basen-Dissoziationskonstanten des Hämoglobins von Menschen-, Pferde- und Rinderblut durch potentiometrische Titration bestimmt. Die erhaltenen, einander entsprechenden Dissoziationskonstanten waren von dem Ursprung des Hämoglobins unabhängig und von der gleicher Grössenordnung.

Die Titrationskurven haben zwei Inflexionspunkte mit Pufferkapazitätsminima, nämlich bei $\mathrm{p}(a \mathrm{H})=5,8$ bzw. 8,7. Die Pufferkapazität des Rinderhämoglobins ist in dem physiologisch wichtigsten Bereich, nämlich zwischen den Inflexionspunkten, kleiner als die des menschlichen und des Pferdehämoglobins. Dieses beruht darauf, dass der Gehalt des Hämoglobins an Säure-Basenfunktionen mit den Dissoziationskonstanten $K_{\mathbf{3}}^{\prime}$ und $K_{\mathbf{3}}^{\prime \prime}$, von denen die Pufferkapazität zwischen den Inflexionspunkten hauptsächlich abhängig ist, beim Rinderhämoglobin kleiner ist als beim Menschen- und Pferdehämoglobin.

Die Titrationskurven des Menschen-, Pferde- und Rinderblutes haben zwei den Inflexionspunkten der Titrationskurve des Hämoglobins entsprechende Inflexionspunkte. Die Pufferkapazität zwischen den Inflexionspunkten vermindert sich in der Reihenfolge: Menschen- $>$ Pferde- $>$ Rinderblut.

Mit Anwendung der erhaltenen Dissoziationskonstanten des Hämoglobins berechnen sich Pufferkapazitätswerte, welche mit Berücksichtigung der Pufferkapazität von Kohlensäure und Phosphorsäure des Blutes mit den experimentell nach der Titrationskurve des Blutes gemessenen Werten übereinstimmen. Die Pufferkapazität des Blutes zwischen den genannten Inflexionspunkten lässt sich hauptsächlich auf das Hämoglobin zurückführen.

Betreffend des Hämoglobins scheint die Pufferwirkung zwischen den Inflexionspunkten hauptsächlich von Hämin und Histidylhistidin des Hämoglobins verursacht zu sein.

Für ein Staatsstipendium zur Förderung der höchsten Geistesbildung sowie für ein Stipendium des Staatlichen Naturwissenschaftlichen Kommission, die mir zur Ausführung der vorliegenden Arbeit bewilligt wurden, bringe ich hier meinen Dank zum Ausdruck.

\section{LITERATUR}

1. Vgl. Errera, J. und Hirshberg, Y. Biochem. J. 27 (1933) 764.

2. Kilpi, S. Sjätte Nordiska Kemistmötet. (1947) 254. Lund.

3. Vgl. Kilpi, S. Z. physik. Chem. A 175 (1935) 240, 248.

4. Kilpi, S. und Meretoja, A. Z. physik. Chem. A 180 (1937) 276.

5. Kilpi, S. Z. physik. Chem. A 180 (1937) 470.

6. Cohn, E. J., Green, A. A. und Blanchard M. H. J. Am. Chem. Soc. 59 (1937) 509. 
7. van Slyke, D. D. J. Biol. Chem. 52 (1922) 522; vgl. Kilpi, S. Z. physik. Chem. A 173 (1935) 224.

8. Kolthoff, I. M.•und Bosch, W. Rec. trav. chim. 47 (1928) 819; Kilpi, S. Z. physik Chem. 173 (1935) 427; Näsänen, R. Acta Chem. Scand. 1 (1947) 204.

9. Hentola, Y. Kemian Keskusliiton julkaisuja 13 (1946) 2.

10. Pauling, L. Proc. Natl. Acad. Sci. U.S. 21 (1935) 186. Ref. nach Cohn ${ }^{6}$.

11. Greenstein, J. P. J. Biol. Chem. 93 (1931) 479.

12. Kilpi, S. und Meretoja, A. Suomen Kemistilehti B 16 (1943) 23.

13. Meretoja, A. Suomalaisen Tiedeakatemian toimituksia A. Chemica. 12 (1944) 28.

Eingegangen am 19. Dezember 1951. 